\title{
Oligometastatic Disease in Upper Gastrointestinal Cancer - How to Proceed?
}

\author{
Costanza Chiapponi Felix Berlth Patrick S. Plum Christopher Betzler Dirk L. Stippel \\ Felix Popp Christiane J. Bruns
}

Department of General, Visceral and Cancer Surgery, University of Cologne, Cologne, Germany

\section{Keywords \\ Oligometastatic disease - Oligometastases . \\ Gastrointestinal cancer}

\section{Summary}

Background: In the present study we review and discuss the current evidence and suggest how to proceed in the management of oligometastatic disease in upper gastrointestinal cancer. Methods: An electronic search of the PubMed database for relevant articles was performed. Results: Both the search for 'oligometastasis', 'oligometastases', 'oligometastatic', 'oligometastatic disease' as well as 'esophageal' and 'esophageal cancer' and the search for 'oligometastasis', 'oligometastases', 'oligometastatic', 'oligometastatic disease' as well as 'gastric', 'gastric cancer', 'stomach', and 'stomach cancer' yielded very few studies. Most data need to be extrapolated in general studies on oligometastatic diseases of different origins. No randomized controlled trial could be found. Conclusion: In the absence of data to formulate recommendations on how to proceed in the treatment of oligometastatic disease in upper gastrointestinal cancer, a more aggressive treatment of oligometastatic disease can be considered in patients whose tumors show a more favorable neoplastic behavior after the 'test of time'. The RENAISSANCE study will certainly deliver important data regarding this aspect.

(c) 2017 S. Karger GmbH, Freiburg

\section{Background}

The concept of 'oligometastatic disease' (OMD) in cancer was first described by Hellman and Weichselbaum [1]. It defines an intermediate stage between limited and metastatic disease, being characterized by the presence of fewer than 5 metastases $[2,3]$. However, synchronous oligometastases (sync-OM) and metachronous oligometastases (oligorecurrence) have a different oncologic significance: the primary site is already controlled in the presence of an oligorecurrence but sync-OM might be the 'tip of the iceberg' [4]. In oligorecurrence, the time between primary treatment and recurrence might give an idea of the tumor biology whereas in sync-OM disease there is no information about the distinct aggressiveness of the malignancy. Another important consideration to take into account is that the evolution of imaging techniques might now even depict extremely early findings; however, it is unclear whether they are ever going to manifest into clinical disease or if they are an early stage of polymetastatic disease. Therefore, it has not been finally clarified if patients really benefit from local treatment strategies for OMD.

There is an increasing number of studies which focus on OMD [5]. The idea behind this growing interest in OMD is the heterogeneity of cancers showing different biological behaviors. Biological cohorts with more favorable prognosis deserve a more aggressive treatment, especially because there are - beside surgical treatments with low mortality and morbidity - very efficient locally effective interventional treatment options such as radiofrequency ablation (RFA) or microwave ablation, brachytherapy, or stereotactic radiation. The first studies on OMD concerned renal cell carcinoma [5]. Meanwhile, surgical resection and interventional ablation of limited hepatic metastases in colorectal cancer, of pulmonary metastases in soft tissue sarcoma, and of brain metastases in non-small-cell lung cancer are common practice worldwide [6]. Concerning upper gastrointestinal cancers, though, there are still controversial opinions on how to proceed in the presence of OMD. This is certainly due to the lack of evidence for these entities. Most available data were generated in retrospective analyses and are consequently not suitable for formulating general recommendations.

In the present study, we review and discuss the current evidence and suggest how to proceed in the management of OMD in upper gastrointestinal cancer.

\section{KARGER}

(C) 2017 S. Karger GmbH, Freiburg

Fax +497614520714 


\section{Methods}

An electronic search of the PubMed database for relevant articles was performed. The following MESH search headings were used: 'oligometastasis', 'oligometastases', 'oligometastatic', 'oligometastatic disease', as well as 'esophageal' and 'esophageal cancer' or 'gastric', 'gastric cancer', 'stomach', and 'stomach cancer'. Reference lists were manually searched for additional articles. Only papers in English and German were included. Beside retrospective studies, review articles and meta-analyses were considered.

\section{Results}

The search 'oligometastasis', 'oligometastases', 'oligometastatic', 'oligometastatic disease', 'esophageal', and 'esophageal cancer' delivered:

- A single-center study on RFA of lung oligometastases from 5 primary lesions including esophageal cancer $(n=123)$ [7]. Patients with esophageal cancer had the worst overall survival.

- A single-center study on stereotactic radiation of metastases 'from any primary site, metastatic to one to three organ sites' [2], in which esophageal cancer patients are included in the group 'non-breast cancer'.

- A recent review complaining about the lack of data regarding this matter [8].

The literature included in the current German Guideline [9] contained two retrospective studies reporting no benefit for resection of metastatic esophageal cancer [10, 11].

The search 'oligometastasis', 'oligometastases', 'oligometastatic', 'oligometastatic disease', 'gastric', 'gastric cancer', 'stomach', and 'stomach cancer' delivered:

- A recent case report with review of the current literature [12].

- A single-center study on the effects of concurrent involved-

field radiotherapy and XELOX on oligometastatic recurrence as opposed to chemotherapy alone [13].

The literature included in the current German Guideline [14] contains a systematic review by Shirabe et al. [15], collecting small retrospective studies on resection of hepatic metastases of gastric cancer.

For both esophageal and gastric cancer, no randomized controlled trial could be found.

\section{Discussion}

According to the guidelines of the European Society for Medical Oncology (ESMO), 'patients with metastatic esophageal cancer can be considered for different options of palliative treatment depending on the clinical situation' [9]. Furthermore, the German guidelines do not recommend esophagectomy in the presence of preoperatively diagnosed metastasis. However, the guidelines consent to resect very limited metastatic disease if this is diagnosed first intraoperatively and if the whole tumor can be surgically resected [9].
Both retrospective studies by Erhunmwunsee et al. [10] and Shimoji et al. [11] are included in the guidelines although resection did not improve the overall survival.

There is no guideline indication concerning the treatment of metachronous distant metastases of esophageal cancer. The decision to resect distant metastases is mostly a personalized treatment recommended by tumor boards for patients in good general condition with a long tumor-free survival. In a small series of 33 patients undergoing pulmonary metastasectomy after definitive treatment of esophageal cancer, however, 70\% developed further distant metastases [16]. Thus, it is unclear if these patients significantly profit from the removal of metastases.

Furthermore, to our knowledge, there are currently no commonly accepted prognostic factors of metastatic esophageal cancer indicating a more favorable biologic behavior except gender. Since (especially premenopausal) women with metastatic squamous esophageal cancer seem to have a lower risk of dying from esophageal cancer when compared with men of similar age [17] and (especially the postmenopausal) show a statistically better prognosis of locoregional advanced disease [17], the female gender might be one of those prognostic factors supporting a more aggressive treatment of these patients.

The situation is similar for metastatic gastric cancer. The present guidelines report a lack of evidence for recommending a curative approach in metastatic gastric cancer. The liver is the most common site for metastases in patients with gastric cancer [18]. The reported rate of liver resection, however, is less than $1 \%$ due to the multiple, bilateral, and extrahepatic nature of the disease $[15,19]$.

The outcome of treatments other than chemotherapy for hepatic metastatic gastric cancer can be mostly pooled out of a larger group called 'non-colorectal, non-neuroendocrine' (NCRNE) in studies. The 5-year survival after surgical R0 resection seems to be as high as $27-37 \%$ [20-22]. In a retrospective study on 257 patients from 5 Japanese centers, however, $75 \%$ of patients had disease recurrence especially in the liver (72.4\%) after a median interval of 7 months after resection [23]. A recent meta-analysis of retrospective studies describes a survival benefit, though [19]. Even in gastric cancer with peritoneal carcinomatosis, maximal cytoreductive surgery combined with regional hyperthermic intraperitoneal and systemic chemotherapy seems to achieve prolonged survival in selected patients [24]. However, a retrospective analysis of 5,185 cases from the SEER database which were initially diagnosed with histologically confirmed gastric cancer with distant metastases between 2004 and 2009 showed that both the resection of only the primary tumor and the combined resection of both the tumors and the metastases were independent prognostic factors for survival improvement. The resection of distant metastases, however, did not significantly improve the survival compared to those patients in which only the primary tumor was removed [25]. In a small study on 35 Chinese patients undergoing both resection of the primary tumor and synchronous hepatic metastatic disease, Liu et al. [26] identified lymphovascular invasion of the primary tumor and multiple liver metastases as negative prognostic factors for survival. 
Kadowaki et al. [27] identified previous gastrectomy (odds ratio $(\mathrm{OR})=1.86 ; \mathrm{p}=0.01)$, single metastatic site $(\mathrm{OR}=1.80 ; \mathrm{p}=0.03)$, and normal alkaline phosphatase levels $(\mathrm{OR}=2.81$; $<<0.01)$ as independent predictors of long-term survival in patients with metastatic gastric cancer undergoing chemotherapy. These might help to identify the subgroup of patients who might benefit of a most aggressive treatment of metastatic gastric cancer [27].

Regarding the significance of liver metastasis in upper gastrointestinal cancer, the meta-analysis by Grimes et al. [19] suggests that patients undergoing ablative treatments of synchronous, multiple, or bilobar metastases have a worse survival compared to those who undergo removal of metachronous singular metastases.

The multicenter FLOT3 study with its three-armed design (in locally advanced tumors, tumors with limited metastases, metastatic tumors of the esophagogastric junction and gastric cancer) suggests that well-selected patients may benefit from surgery at the stage of limited metastases [28]. However, there are currently no prognostic factors or biomarkers to identify which patients will benefit.

The FLOT5 study (RENAISSANCE) examines the effect of chemotherapy alone versus chemotherapy followed by surgical resection on survival and quality of life in patients with limited metastatic adenocarcinoma of the stomach or esophagogastric junction. First results are expected in 2021.

\section{Conclusions}

Several retrospective analyses suggest that long-term survival is 'better than expected' after ablative treatment of OMD in upper gastrointestinal cancers. However, this effect might not be due to the treatment strategies themselves but rather due to the selection of patients [29].

There is general agreement that a metachronous isolated oligometastatic relapse in patients with good systemic therapy response can be surgically or interventionally removed. At the moment, the 'test of time' is the most convincing indicator of a more favorable biological cancer behavior. However, there is an increasing shift toward individualized, multidisciplinary management of OMD because it is difficult to conduct randomized controlled trials due to the variety of presentations [5].

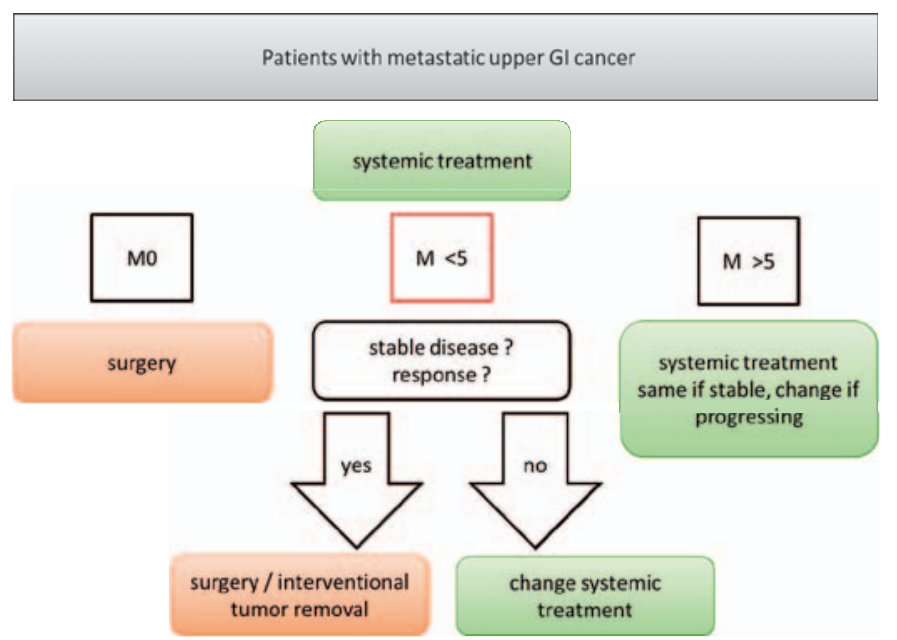

Fig. 1. Therapeutic management of oligometastatic disease in upper gastrointestinal cancer. The 'test of time' and the response to systemic treatment are currently the only useful markers for a more favorable and less aggressive biological behavior of the disease and encourage to pursue a more aggressive treatment.

In the absence of prognostic factors for metastatic upper gastrointestinal disease, biomarkers indicating which patients might benefit from a more aggressive surgical or radiological removal of OMD are probably the key to this problem. The presence of circulating tumor cells, for example, seems to correlate with disease progression and survival [30]. Moreover, serum microRNAs could potentially be useful biomarkers [31]. Examples of pro-metastatic microRNAs include microRNA-10b (upregulated in primary metastatic breast cancer) and microRNA-21 (correlates with advanced stage and poor outcomes in breast and pancreatic cancer) [5].

The 'test of time' in patients with good systemic response might warrant a more aggressive ablative treatment of OMD even in upper gastrointestinal cancer (fig. 1). Until more data are available, OMD in upper gastrointestinal cancer should be managed with an individualized, multidisciplinary approach.

\section{Disclosure Statement}

The authors have no conflicts of interests.

\section{References}

1 Hellman S, Weichselbaum RR: Oligometastases. J Clin Oncol 1995;13:8-10.

2 Milano MT, Katz AW, Zhang H, Okunieff P: Oligometastases treated with stereotactic body radiotherapy: long-term follow-up of prospective study. Int J Radiat Oncol Biol Phys 2012;83:878-886.

3 Weichselbaum RR, Hellman S: Oligometastases revisited. Nat Rev Clin Oncol 2011;8:378-382.

4 Yamashita H, Niibe Y, Yamamoto T, Katsui K, Jingu K, Kanazawa S, Terahara A, Nakagawa K: Lung stereotactic radiotherapy for oligometastases: comparison of oligo-recurrence and sync-oligometastases. Jpn J Clin Oncol 2016;46:687-691.
5 Reyes D: The biology and treatment of oligometastatic cancer. Oncotarget 2015;6:8491-8524.

6 Broomfield JA, Greenspoon JN, Swaminath A: Utilization of stereotactic ablative radiotherapy in the management of oligometastatic disease. Curr Oncol 2014; 21:115-117.

7 Omae K, Hiraki T, Gobara H, Iguchi T, Fujiwara H, Matsui Y, Toyooka S, Nagasaka T, Kanazawa S: Longterm survival after radiofrequency ablation of lung oligometastases from five types of primary lesions: a retrospective evaluation. J Vasc Interv Radiol 2016;27: $1362-1370$.
8 Behrens A, Ell C, Lordick F: Perioperative and palliative chemotherapy for esophageal cancer. Viszeralmedizin 2015;31:341-346.

9 Lordick F, Mariette C, Haustermans K, Obermannová R, Arnold D; on behalf of the ESMO Guidelines Committee: Oesophageal cancer: ESMO Clinical Practice Guidelines for diagnosis, treatment and follow-up. Ann Oncol 2016;27(suppl 5):v50-v57.

10 Erhunmwunsee, L, Englum BR, Onaitis MW, D’Amico TA, Berry MF: Impact of pretreatment imaging on survival of esophagectomy after induction therapy for esophageal cancer: who should be given the benefit of the doubt?: esophagectomy outcomes of patients with suspicious metastatic lesions. Ann Surg Oncol 2015;22: 1020-1025. 
11 Shimoji, H, Karimata H, Nagahama M, Nishimaki T: Induction chemotherapy or chemoradiotherapy followed by radical esophagectomy for T4 esophageal cancer: results of a prospective cohort study. World J Surg 2013;37:2180-2188.

12 Negoi I, Runcanu A, Paun S, Negoi RI, Beuran M: Resection of large metachronous liver metastasis with gastric origin: case report and review of the literature. Cureus 2016;8:e814.

13 Xu C, Xie J, Liang N, Wang J, Qiao L, Luo H, Zhang JX, Zhang J: Concurrent involved-field radiotherapy and XELOX in gastric cancer patients with postoperative oligometastatic recurrence. J Cancer Res Ther 2014 10(suppl):267-271.

14 Moehler M, Al-Batran S-E, Andus T, et al: German S3guideline 'Diagnosis and treatment of esophagogastric cancer' (Article in German). Z Gastroenterol 2011;49: 461-531.

15 Shirabe K, Wakiyama S, Gion T, Watanabe M, Miyazaki $M$, Yoshinaga $K$, Tokunaga $M$, Nagaie T: Hepatic resection for the treatment of liver metastases in gastric carcinoma: review of the literature. HPB (Oxford) 2006;8:89-92.

16 Kanamori J, Aokage K, Hishida T, Yoshida J, Tsuboi M, Fujita T, Nagino M, Daiko H: The role of pulmonary resection in tumors metastatic from esophageal carcinoma. Jpn J Clin Oncol 2017;47:25-31.

17 Bohanes P, Yang D, Chhibar RS, Labonte MJ, Winder T, Ning Y, Gerger A, Benhaim L, Paez D, Wakatsuki T, Loupakis F, El-Khoueiry R, Zhang W, Lenz HJ: Influence of sex on the survival of patients with esophageal cancer. J Clin Oncol 2012;30:2265-2272.

18 Riihimäki M, Hemminki A, Sundquist K, Sundquist J, Hemminki K: Metastatic spread in patients with gastric cancer. Oncotarget 2016;7:52307-52316.
19 Grimes N, Devlin J, Dunne DFJ, Poston G, Fenwick S, Malik H: The role of hepatectomy in the management of metastatic gastric adenocarcinoma: a systematic review. Surg Oncol 2014;23:177-185.

20 Takemura N, Saiura A, Koga R, Arita J, Yoshioka R, Ono Y, Hiki N, Sano T, Yamamoto J, Kokudo N, Yamaguchi T: Long-term outcomes after surgical resection for gastric cancer liver metastasis: an analysis of 64 macroscopically complete resections. Langenbecks Arch Surg 2012;397:951-957.

21 Markar SR, Mikhail S, Malietzis G, Athanasiou T, Mariette C, Sasako M, Hanna GB: Influence of surgical resection of hepatic metastases from gastric adenocarcinoma on long-term survival: systematic review and pooled analysis. Ann Surg 2016;263:1092-1101.

22 Petrelli F, Coinu A, Cabiddu M, Ghilardi M, Borgonovo K, Lonati V, Barni S: Hepatic resection for gastric cancer liver metastases: a systematic review and meta-analysis. J Surg Oncol 2015;111:1021-1027.

23 Kinoshita T, Kinoshita T, Saiura A, Esaki M, Sakamoto $\mathrm{H}$, Yamanaka T: Multicentre analysis of long-term outcome after surgical resection for gastric cancer liver metastases. Br J Surg 2015;102:102-107.

24 Rudloff U, Langan RC, Mullinax JE, Beane JD, Steinberg SM, Beresnev T, Webb CC, Walker M, Toomey MA, Schrump D, Pandalai P, Stojadinovic A, Avital I: Impact of maximal cytoreductive surgery plus regional heated intraperitoneal chemotherapy (HIPEC) on outcome of patients with peritoneal carcinomatosis of gastric origin: results of the GYMSSA trial. J Surg Oncol 2014;110:275-284.

25 Chen JQ, Kong YY, Weng SS, Dong CX, Zhu LZ, Yang Z, Zhong J, Yuan Y: Outcomes of surgery for gastric cancer with distant metastases: a retrospective study from the SEER database. Oncotarget 2016;DOI: 10.18632/oncotarget.14027.
26 Liu Q, Bi JJ, Tian YT, Feng Q, Zheng ZX, Wang Z: Outcome after simultaneous resection of gastric primary tumour and synchronous liver metastases: survival analysis of a single-center experience in China Asian Pac J Cancer Prev 2015;16:1665-1669.

27 Kadowaki S, Komori A, Takahari D, Ura T, Ito S, Tajika M, Niwa Y, Oze I, Muro K: Clinical characteristics associated with long-term survival in metastatic gastric cancer after systemic chemotherapy. Asian Pac J Cancer Prev 2015;16:5433-5438.

28 Sunan K, Hofheinz R, Homann N, Illerhaus G, Mönig SP, Pauligk C, Jäger E, Kraus TW, Al Batran SE; Arbeitsgemeinschaft Internistische Onkologie (AIO): Gibt es Kandidaten für eine bimodale Behandlungsstrategie beim metastasierten Magenkarzinom? - Endergebnisse der prospektiven multizentrischen FLOT3-Studie der AIO. 130. Kongress der Deutschen Gesellschaft für Chirurgie. München. 2013 Meeting Abstract;DOI: 10.3205/13dgch152.

29 Palma D, Salama JK, Lo SS, Senan S, Treasure T, Govindan $\mathrm{R}$, Weichselbaum $\mathrm{R}$ : The oligometastatic state - separating truth from wishful thinking. Nat Rev Clin Oncol 2014;11:549-557.

30 Tanaka M, Takeuchi H, Hiraiwa K, Nakamura R, Takahashi T, Wada N, Kawakubo H, Saikawa Y, Omori T, Kitagawa Y: Clinical significance of circulating tumor cells in blood from patients with advanced or recurrent esophageal cancer. J Clin Oncol 2012;30 (suppl):19.

31 Plum PS, Bollschweiler E, Hölscher AH, WarneckeEberz U: Novel diagnostic and prognostic biomarkers in esophageal cancer. Expert Opin Med Diagn 2013;7: 557-571. 